\title{
A New Remote Authentication Scheme for Anonymous Users Using ECC
}

\author{
Dianli Guo \\ School of Mathematical Sciences \\ University of Jinan \\ Jinan 250022, China \\ e-mail: guodianli@163.com
}

\author{
Fengtong Wen \\ School of Mathematical Sciences \\ University of Jinan \\ Jinan 250022, China \\ e-mail: wftwq@163.com
}

\begin{abstract}
In 2011, Khan et al. identified that Wang et al. scheme could not achieve user anonymity and had many practical pitfalls, In addition, Khan et al. presented an enhanced scheme to eliminate the aforementioned defects. In this article, we point out that Khan et al.'s scheme is incapable to provide user anonymity and suffers from forgery attack, offline password guessing attack. Besides, a new authentication scheme for anonymous users using elliptic curves cryptosystem is presented, which could withstand various types of network attacks and is more suitable for mobile application scenarios where resources constrained and security concerned.
\end{abstract}

Keywords- anonymity; authentication; ID-based; ECC

\section{INTRODUCTION}

It is necessary for service providing servers to authenticate remote users when users access resources through public network; remote authentication is an essential mechanism for them to authenticate each other conveniently. However, a majority of the solutions are susceptible to a range of potential security attacks.

Recently, dozens of anonymous authentication schemes $[3,4,5,6,7,8]$ which emphasized the security problems were proposed. However, a majority of them were demonstrated to be insecure for the augmented reality applications.

In 2011, Khan et al. [9] proposed a remote user authentication scheme which was claimed to preserve user anonymity. However, we investigate that it is insecure. Further, user anonymity is neglected by authors. In order to conquer the aforementioned flaws, a new scheme preserving user privacy is presented.

This paper is organized as follows. In section II and section III, we presented the brief review of Khan et al.'s scheme and describe the weaknesses of their scheme, respectively. In section IV, we propose an authentication scheme. The analysis of our proposal and the comparisons with other related schemes are presented in section V. At last, section VI concludes this paper.

\section{OVERVIEW OF KHAN ET AL.'S SCHEME}

We will present the brief review the scheme of [9] in this section.

\section{A. Registration phase}

Step1. $U_{i}$ chooses $I D_{i}, P W_{i}$ and a nonce $r$. Then $U_{i}$ computes $R P W=H\left(r \square P W_{i}\right)$ and sends $I D_{i}$, $R P W$ to $S$ for registration.

Step2. $S$ checks the registration credential. If the received $I D_{i}$ is already stored in the database, $U_{i}$ has to choose another identity. More over, $S$ checks the registration record for $U_{i}$. Assume that $U_{i}$ is a new user, $S$ will set the value $N=0$; otherwise, $S$ will set $N=1$ and store $I D_{i}$ with $N$ in the database.

Step3. $S$ computes $I D U=\left(I D_{i} \| N\right), J=H(x$ II $I D U)$ and $L=J \oplus R P W$. Then $S$ sends the smart card to $U_{i}$ containing $\{L, y\}$ where $y$ is the shared secret key.

Step4. $U_{i}$ stores $r$ into the received smart card.

\section{B. Login phase}

Step1. $U_{i}$ inputs $I D_{i}, P W_{i}$. Then the smart card computes $R P W=H\left(r \| P W_{i}\right) \quad, \quad J=L \oplus R P W \quad$, $C_{1}=H\left(T_{i} \| J\right) \quad$ and $\quad A I D_{i}=I D_{i} \oplus H\left(y_{\|}^{\square} T_{i} d\right)$ where $d$ is a nonce and $T_{i}$ is the fresh timestamp.

Step2. $\left\{A I D_{i}, T_{i}, d, C_{1}\right\}$ is transmitted to $S$.

\section{Authentication phase}

Step1. Upon receiving the login request at $T_{i}^{\prime}, S$ checks the time interval by verifying $T_{i}^{\prime}-T_{i} \leq \Delta T$. If the verification fails, $S$ terminates this procedure.

Step2. $S$ computes $I D_{i}=A I D_{i} \oplus H\left(y \eta_{\|} T_{i} \| d\right)$ and verifies its validity. If $I D_{i}$ is valid, executes step 3 ; otherwise, $S$ rejects this request.

Step3. $S$ retrieves $N$ from the database with $I D_{i}$ and computes $I D U=\left(I D_{i} \prod_{\|} N\right), J=H\left(x \|_{\|} I D U\right)$. Then 
$S$ checks whether $C_{1}$ ? $=H\left(T_{i} \square J\right)$. If the equality holds, $S$ will accept the login request and $U_{i}$ is authentic.

Step4. $S$ computes $C_{2}=H\left(C_{1} \oplus J \oplus T_{s}\right)$, where $T_{s}$ is the fresh timestamp and then transmits the reply message $\left\{C_{2}, T_{s}\right\}$ to $U_{i}$.

Step5. On receiving the reply message at $T_{s}^{\prime}, U_{i}$ verifies the freshness of $T_{s}$. If $T_{s}^{\prime}-T_{s} \geq \Delta T, U_{i}$ terminates this operation. Then $U_{i}$ checks $H\left(C_{1} \oplus J \oplus T_{s}\right) \quad$ ? $=C_{2}$. If fails, this session is terminated.

Step6. At the end, $U_{i}$ and $S$ compute and share the session key $S K=H\left(C_{2} \oplus J\right)$ for the further communication.

\section{Password-change phase}

Step1. $U_{i}$ enters $I D_{i}$ and $P W_{i}$ to the smart card and computes $R P W^{*}=h\left(r \square P W_{i}\right)$ and $J^{*}=L \quad \oplus P W^{*}$. If $J^{*}=J, U_{i}$ inputs a new password $P W_{i}^{\text {new }}$; otherwise, this procedure is terminated.

Step2: Computes $L^{\text {new }}=L \oplus R P W \oplus H\left(r \square P W_{i}^{\text {new }}\right)$ and stores $L^{\text {new }}$ into its memory to replace $L$.

\section{E. Revocation phase}

Smart cards may be lost or stolen, $S$ should allow the cardholder $U_{i}$ to execute the revocation. $S$ firstly verifies the validity of $U_{i}$ 's credential as mentioned in the registration phase. After that, $S$ changes $N$ to be $N+1$. Finally, $S$ performs the same steps in the registration phase with another $N$.

\section{CRYPTANALYSIS OF KHAN ET AL.'S SCHEME}

We analyze the security of Khan et al.'s scheme and show their scheme is incapable to resist attacks. More over, the feature of user anonymity is not preserved in their scheme.

\section{A. No provision of users anonymity}

The user' specific and secret information (e.g., the login history and the current location) may be obtained by a malicious adversary due to the leakage of user's static identity. However, Khan et al.'s scheme [9] cannot achieve user anonymity.

Step1. Any legal but anonymous user $U_{k}$ can obtain the secret value $y$ from his/her smart card.
Step2. When $U_{i}$ logins to $S, U_{k}$ can intercept his/her login request $\left\{A I D_{i}, T_{i}, d, C_{1}\right\}$. Then $U_{k}$ can compute $I D_{i}=A I D_{i} \oplus H\left(y \square T_{i} \square d\right)$.

\section{B. Off-line password guessing attack}

Suppose that the smart card of a legal remote user $U_{i}$ is stolen or picked up by $U_{k}$, and the data $\{L, y, r\}$ in the smart card [1,2]. Then $U_{k}$ can perform the following procedures to get $U_{i}$ 's password $P W_{i}$ :

Step1. Choose a password $P W_{i}^{*}$.

Step2. Computes $C_{1}^{*}=H\left(T_{i} \square\left(L \oplus H\left(r \square P W_{i}^{*}\right)\right)\right.$ and checks $C_{1}^{*}$ ? $=C_{1}$ to confirm the correctness of $P W_{i}^{*}$.

Step3. Repeats the step 1 and 2 by replacing another guessed password until the correct password is found.

\section{Forgery attack}

As explained above, with the extracted values $\{L, y, r\}$ [1,2], $U_{k}$ can get $P W_{i}$ and $I D_{i}$ corresponding to $U_{i}$. And he/she can further impersonate $U_{i}$ to deceive $S$ by performing the following steps.

Step1. Computes $R P W=H\left(r \square P W_{i}\right), \quad J=L$ $\oplus R P W \quad, \quad C_{1}^{\prime}=H\left(T_{k} \square J\right), \quad A I D_{i}^{\prime}=I D_{i} \oplus H(y$ $\square T_{k} \square d^{\prime}$ ), where $T_{k}$ is the fresh current time stamp and $d^{\prime}$ is a nonce generated by $U_{k}$, then, sends the forged login request message $\left\{A I D_{i}^{\prime}, T_{k}, d^{\prime}, C_{1}^{\prime}\right\}$ to $S$.

Obviously, $S$ can accept the login request of the adversary, since these parameters of the forged login request message are in the correct format. Thus, the adversary can successfully impersonate as $U_{i}$ to communicate with $S$.

\section{OUR Proposed SCHEME}

We propose an anonymous authentication scheme in this section.

\section{A. Registration phase}

Initially, $S$ chooses two distinct large primes $p$ and $q$ with $p=2 q+1$. $E_{p}(a, b)$ is an elliptic curve in the finite field $Z_{p}$. $P$ is a generator of order $q$ on the elliptic curve $E_{p}(a, b) . S$ computes $Q=x \cdot P \bmod p$ where $x$ is the master secret key of $S .\left\{P, p, Q, E_{p}(a, b)\right\}$ are the public parameters. 
Step1. $U_{i}$ selects his/her identity $I D_{i}$ and password $P W_{i}$, then sends them to $S$ over a secure channel.

Step2. Upon receiving the message $\left\{I D_{i}, P W_{i}\right\}, S$ computes $A_{i}=H\left(I D_{i} \| P W_{i}\right)$ and $B_{i}=H\left(I D_{i} \cdot y \cdot Q\right)$ $\oplus H\left(I D_{i} \oplus P W_{i}\right)$, where $y$ is a nonce chosen by $S$ for every user.

Step3. $S$ issues the smart card stored $\left\{A_{i}, B_{i}, p, E_{p}(a, b), P, Q, H(\cdot)\right\}$ to $U_{i}$.

\section{B. Login phase}

Step1. $U_{i}$ keys $I D_{i}^{*}, P W_{i}^{*}$. The smart card computes $A_{i}^{*}=H\left(I D_{i}^{*} \| P_{i}^{*}\right)$ and verifies $A_{i}^{*} ?=A_{i}$. If yes, it means $U_{i}$ is the cardholder; otherwise, login request is terminated.

Step2. The smart card generates a random nonce $d$ and computes $H\left(I D_{i} \cdot y \cdot Q\right)=B_{i} \oplus H\left(I D_{i} \oplus P W_{i}\right), C_{i}$ $=I D_{i} \cdot P+d \cdot Q, D_{i}=d \cdot P, E_{i}=H\left(I D_{i} \cdot y \cdot Q\right) \cdot P$ $+d \cdot T_{i} \cdot Q$, where $T_{i}$ is the fresh current time stamp.

Step3. After that, $U_{i}$ sends $\left\{C_{i}, D_{i}, E_{i}, T_{i}\right\}$ to $S$.

C. Authentication phase

Step1. Upon receiving $\left\{C_{i}, D_{i}, E_{i}, T_{i}\right\}$ at $T_{i}^{\prime}, S$ verifies the freshness of $T_{i}$, if $T_{i}^{\prime}-T_{i} \leq \Delta T$ holds, $S$ continues the next step.

Step2. Afterwards, $S$ calculates $I D_{i} \cdot P=C_{i}-x \cdot D_{i}$ and $E_{i}^{*}=H\left(x \cdot y \cdot I D_{i} \cdot P\right) \cdot P+x \cdot T_{i} \cdot D_{i}$. If the digest value of $E_{i}^{*}$ equals to received $E_{i}$, proceeds to step 3 ; else, this session will be terminated.

Step3. $S$ computes $F_{i}=\mathbb{D}_{i} \cdot P+r \cdot P, G_{i}=r \cdot I_{i} \cdot P+T_{s} \cdot x \cdot D_{i}$. Then, $S$ transmits the replied message $\left\{F_{i}, G_{i}, T_{s}\right\}$ to $U_{i}$.

Step4. The smart card verifies the freshness of $T_{s}$. Subsequently, computes $r \cdot P=F_{i}-I D_{i} \cdot P$ and checks whether $G_{i}$ equals to the computed $I D_{i} \cdot r \cdot P+T_{s} \cdot d \cdot Q$. If yes, $S$ is authentic and the authentication is completed successfully.

After that, $U_{i}$ and $S$ agree on the session key $S K=d \cdot r \cdot P$.

C. Password changing phase

Step1. The smart card performs step 1 of the login phase.
Step2. If $U_{i}$ is the cardholder, $U_{i}$ inputs a new password $P W_{i}^{\text {new }}$; on the contrary, the smart card terminates the request of updating the password.

Step3. The smart card calculates $A_{i}^{\text {new }}$ $=H\left(I D_{i} \| P W_{i}^{\text {new }}\right) \quad, \quad B_{i}^{\text {new }}=B_{i} \oplus H\left(I D_{i} \oplus P W_{i}\right)$ $\oplus H\left(I D_{i} \oplus P W_{i}^{\text {new }}\right)$ and stores $A_{i}^{\text {new }}, B_{i}^{\text {new }}$ into the smart card to replace $A_{i}, B_{i}$.

\section{SECURITY ANALySIS OF OUR SCHEME}

\section{A. Preserving user anonymity}

If $U_{k}$ has intercepted all of $U_{i}$ 's authentication messages $\left\{C_{i}, D_{i}, E_{i}, F_{i}, G_{i}, T_{i}, T_{s}\right\}$. There is no any static value in these parameters based on the randomness of $d$ and $r$. Accordingly, $U_{k}$ cannot retrieve $I D_{i} \cdot P$ from $C_{i}$ without knowing the random numbers of $d$ and $r$ since the difficulty of the Computation Diffie-Hellman problem.

\section{B. Off-line password guessing attack}

Suppose that $U_{k}$ has gotten smart card of another legitimate user $U_{i}$ and the value $A_{i}$ can be revealed from the smart card. After that $U_{k}$ may try to guess both $I D_{i}$ and $P W_{i}$ by computing $A_{i}=H\left(I D_{i} \| P W_{i}\right)$, because we have demonstrated that user anonymity confidentiality is achieved in the proposed scheme. It is impossible for the adversary to guess identity and password correctly simultaneously in polynomial time.

\section{Forgery attack}

$U_{k}$ should attempts to forge a valid login request $\left\{C_{i}, D_{i}, E_{i}, T_{i}\right\}$. However, $U_{k}$ cannot compute the $C_{i}$ and $E_{i}$ without knowing $I D_{i}$ and the secret number $y$ generated by $S$, even if $U_{k}$ has extracted the secret values $\left\{A_{i}, B_{i}, p, E_{p}(a, b), P, Q, H(\cdot)\right\}$ stored in $U_{i}$ 's smart card. Hence, $U_{k}$ cannot launch the forgery attack to fool the server.

\section{Server impersonating attack}

In order lunch the server spoofing attack to fool $U_{i}, U_{k}$ may attempt to forge a legal reply message $\left\{F_{i}, G_{i}, T_{s}\right\}$ after receiving $U_{i}$ 's login request message. However, $U_{k}$ cannot compute the forged reply message without knowing the master secret key $x$ of the server, even if $U_{k}$ has extracted the secret data from $U_{i}$ 's smart card. Hence, $U_{k}$ 
cannot masquerade as $S$ to fool $U_{i}$ and the server spoofing attack is meaningless in the proposed scheme.

\section{E. Comparison}

In this section, the security features comparison of Ma et al.'s [8] and Khan et al.'s [9] schemes is presented to evaluating our scheme. We summarize the detailed comparisons in the Table II. As you can see in Table II, our proposal is relatively more secure than the other two schemes.

TABLE I. TABLE TyPE STYLES

\begin{tabular}{|c|c|c|c|}
\hline & Khan et al.[9] & Ma et al. [8] & Ours \\
\hline User anonymity & No & No & Yes \\
\hline Prevention of forgery attack & No & No & Yes \\
\hline Prevention of off-line dictionary attack & No & No & Yes \\
\hline Prevention of server spoofing attack & Yes & No & Yes \\
\hline Freely change password & Yes & Yes & Yes \\
\hline Mutual authentication & Yes & Yes & Yes \\
\hline
\end{tabular}

\section{CONCLUSION}

In this paper, we find out that Khan et al.'s scheme is insecure and incapable to provide user privacy. Also, we proposed a robust anonymous authentication scheme using elliptic curves cryptosystem to conquer the aforementioned weaknesses. Security analysis show that our proposal is relatively more secure than the related schemes in terms of the security.

\section{REFERENCES}

[1] P. Kocher, J. Jaffe and B. Jun, "Differential power analysis," 19th Annual international cryptology conference, 1999, p. 388-97.

[2] T. S. Messerges, E. A. Dabbish and R. H. Sloan, "Examining smart card security under the threat of power analysis attacks," IEEE Trans Comput, 2002, 5(51):541-552.

[3] M. L. Das, A. Saxena and V. P. Gulati, "A dynamic ID-based remote user authentication scheme," IEEE Trans on Consumer Electronics, 2004, 50(2):665-667.

[4] Y. F. Chang and P. Y. Chang, "An improved user authentication and key agreement scheme providing user anonymity," Journal of Electronic Science and Technology, 2011, 4(9):352-358.

[5] Y. Y. Wang, J. Y. Liu, F. X. Xiao and J. Dan, "A more efficient and secure dynamic ID-based remote user authentication scheme," Computer Communications, 2009, 4(32):583-585.

[6] F. T. Wen and X. L. Li, "An improved dynamic ID-based remote user authentication with key agreement scheme," Computers and Electrical Engineering, 2011, 38(2):381-387.

[7] R. C. Wang, W. S. Juang and C. L. Lei, "Robust authentication and key agreement scheme preserving the privacy of secret key," Computer Communications, 2011, 3(34):274-280.

[8] Y. Ma, G. W. Li and L. M. Zhang, "A Novel Remote Authentication Scheme Based-On Password for Anonymous Users," Information and Business Intelligence, 2012, 187-194.

[9] M. K. Khan, S. K. Kim and K. Alghathbar, "Cryptanalysis and security enhancement of a 'more efficient \& secure dynamic ID-based remote user authentication scheme," Computer Communications, 2011, 34(3):305-309. 